\title{
Predictive value of angiogenic factors, clinical risk factors and uterine artery Doppler for pre- eclampsia and fetal growth restriction in second and third trimester pregnancies in an Ecuadorian population
}

Carolin Kienast, Walter Moya, Oswaldo Rodriguez, Alfredo Jijón \& Annegret Geipel

To cite this article: Carolin Kienast, Walter Moya, Oswaldo Rodriguez, Alfredo Jijón \& Annegret Geipel (2016) Predictive value of angiogenic factors, clinical risk factors and uterine artery Doppler for pre-eclampsia and fetal growth restriction in second and third trimester pregnancies in an Ecuadorian population, The Journal of Maternal-Fetal \& Neonatal Medicine, 29:4, 537-543, DOI: 10.3109/14767058.2015.1012063

To link to this article: http://dx.doi.org/10.3109/14767058.2015.1012063

Published online: 24 Feb 2015.

Submit your article to this journal $\longleftarrow$

山 Article views: 80

Q View related articles $\sqsubset$

View Crossmark data ד 


\title{
Predictive value of angiogenic factors, clinical risk factors and uterine artery Doppler for pre-eclampsia and fetal growth restriction in second and third trimester pregnancies in an Ecuadorian population
}

\author{
Carolin Kienast ${ }^{1}$, Walter Moya ${ }^{2}$, Oswaldo Rodriguez ${ }^{3}$, Alfredo Jijón ${ }^{4}$, and Annegret Geipel ${ }^{5}$ \\ ${ }^{1}$ Department of Obstetrics and Gynecology, Marienhaus Klinikum St. Elisabeth, Neuwied, Bonn, Germany, ${ }^{2}$ Faculty of Medicine, Universidad Central \\ del Ecuador, Quito, Ecuador, ${ }^{3}$ Roche Diagnostics Ecuador, Quito, Ecuador, ${ }^{4}$ Faculty of Medicine, Pontificia Universidad Catolica del Ecuador, Quito, \\ Ecuador, and ${ }^{5}$ Department of Obstetrics and Prenatal Medicine, University of Bonn, Bonn, Germany
}

\section{Abstract}

Objective: To evaluate the performance of angiogenic factors, maternal risks and uterine artery Doppler (UAD) in the prediction of pre-eclampsia (PE) and fetal growth restriction (FGR) in a high-risk Ecuadorian population.

Methods: Patients with singleton pregnancies $(n=346)$ were investigated at two clinical visits (18-25 weeks and 28-32 weeks). Mean uterine artery (UA), pulsatility index (PI) and maternal biomarkers (soluble fms-like tyrosine kinase-1, placental growth factor, sFIt-1/PLGF ratio) were obtained. The main endpoints were PE and FGR. UA PI and angiogenic factor levels were compared for the groups with PE $(n=34)$, FGR $(n=26)$, PE \& FGR $(n=14)$ and controls $(n=272)$. Multivariable stepwise logistic regression was used to construct prediction models. Results: Pregnancies with either FGR or PE \& FGR exhibited in the second trimester a significantly higher mean UA PI and sFlt-1/PLGF ratio and lower PLGF values compared to controls. In the third trimester, all groups with adverse outcome demonstrated significantly lower PLGF levels and a higher sFlt-1/PLGF ratio compared to normal pregnancies. Differences were most pronounced for pregnancies that developed PE and FGR for both time intervals. The combination of UAD and sFlt-1/PLGF ratio improved the predictive capacity for PE and FGR compared to each parameter alone. The best performance was obtained by integrating anamnestic risk factors, resulting in an area under the receiver operating curve for PE of 0.85 and 0.89 and for FGR of 0.79 and 0.77 in the second and third trimester, respectively.

Conclusion: In a high-altitude Ecuadorian population, angiogenic factors and UA PI were useful tools in the prediction of PE and/or FGR. The highest performance was achieved by the combination of these factors, including obstetric and medical history.
\end{abstract}

\section{Keywords}

Angiogenic factors, Ecuador, fetal growth restriction, high altitude, placental growth factor, pre-eclampsia, soluble fms-like tyrosine kinase-1, uterine artery Doppler

\section{History}

Received 22 October 2014

Revised 18 January 2015

Accepted 22 January 2015

Published online 24 February 2015

\section{Introduction}

Pre-eclampsia (PE) is a pregnancy-specific multisystem disorder that affects $2-8 \%$ of women worldwide. It contributes substantially to fetal and maternal morbidity and mortality, especially in developing countries [1].

PE and fetal growth restriction (FGR) share several mechanisms of disease which include disturbed uteroplacental perfusion with chronic ischemia and various endothelial cell dysfunction. There is increasing evidence that the imbalance between pro-angiogenic factors such as placental growth factor (PLGF) and the anti-angiogenic factor soluble fms-like tyrosine kinase-1 (sFlt-1) participate in the pathophysiology of both, PE and FGR. However, it is still unclear why some

Address for correspondence: Prof. Annegret Geipel, Department of Obstetrics and Prenatal Medicine, University Medical School Bonn, Sigmund-Freud-Str. 25, 53105 Bonn, Germany. Tel: +49 22828715942. Fax: +49 228287 16088. E-mail: annegret.geipel@ukb.uni-bonn.de women with similar findings develop PE, while others develop FGR and some may be affected of both [2-4].

A number of second and third trimester studies demonstrated that pre-eclamptic patients present significantly higher levels of sFlt-1 accompanied by significantly lower levels of PLGF compared to controls. It has been shown that altered maternal sFlt-1 and PLGF levels are detectable weeks before the onset of PE and FGR, but absolute levels varied widely between studies [5-10]. More recently, the sFlt-1/PLGF ratio has been proposed as an aid to enhance the diagnostic accuracy of screening [11]. Further, abnormal uterine artery Doppler (UAD) is a well-known surrogate marker of disturbed placental development. Increased UAD resistance and/or early diastolic notching in the second trimester equally are associated with an elevated risk for PE and FGR through the course of pregnancy [12-14]. In some studies, angiogenic markers combined with UAD investigation had more predictive ability than any of these examinations alone $[15,16]$. 
In the highlands of Ecuador $(2850 \mathrm{~m})$, the prevalence of PE is between $12 \%$ and $15 \%$, which is up to 4 times higher in comparison to its prevalence in low-altitude residents. Compared with babies born at low-altitude, high-altitude babies in Bolivia have a greater occurrence of FGR (17\% versus 6\%). While the reason behind this has not been fully studied yet, current hypothesis constitute a relationship to the high altitude. In addition, pre-eclamptic women show an overexpression of placental hypoxia-inducible factor (HIF), which is also increased in high-altitude residents [17-19].

Especially in countries with limited resources but a high prevalence, timely identification of women at risk is vital in order to adjust prenatal care. Consequently, the aim of this study was to investigate the usefulness of sFlt-1 and PLGF determination in combination with uterine artery (UA) Doppler for the prediction of PE and FGR in second and third trimester Ecuadorian pregnancies.

\section{Methods}

\section{Study design}

The study was designed as a prospective cohort study and included 346 pregnant women who attended the routine obstetrical care at Hospital Isidro Ayora in Quito, Ecuador, between April and December 2010. Approval of the local ethics committee was provided. All women gave written, informed consent. Patients with singleton pregnancies were enrolled from the second trimester of pregnancy (first visit between 18 and 25 weeks) to their discharge after delivery.

At recruitment and after delivery, all women underwent a standard workup including previous and current obstetric history, blood pressure determination and quantification of urine protein excretion by dipstick. Routine ultrasound investigation included fetal biometry, exclusion of major malformations and UAD studies. UAD examinations were performed transabdominally using standardized criteria [15]. The pulsatility index (PI) was calculated as a mean from both sides and the presence or absence of a notching was noted. There was one principle investigator (C.K.). There was a second time interval of examination between 28 and 32 weeks of gestation, which all women attended.

At each of the two visits, maternal venous blood samples $(10 \mathrm{ml})$ were collected into tubes containing EDTA. Within $2 \mathrm{~h}$, the plasma was separated by centrifugation at $4000 \mathrm{~g}$ for $10 \mathrm{~min}$ and stored at $-80^{\circ} \mathrm{C}$ until testing. Maternal plasma sFlt- 1 and PLGF concentration were measured using a commercial Roche Elecsys System (Rotkreuz, Switzerland) (Elecsys PLGF, human PLGF, and Elecsys sFlt-1, human sFlt-1). For each sample, the sFlt-1/PLGF ratio was calculated.

Optimal cutoffs were calculated based on multifactorial receiver operator characteristics. The cutoff was set as optimal when the classification rate was optimal, that is, the sum of sensitivity and specificity was maximal.

The main endpoints were PE and FGR. PE was defined as new-onset proteinuric hypertension after 20 weeks of pregnancy; hypertension as either a systolic blood pressure $\geq 140 \mathrm{mmHg}$ or a diastolic blood pressure $\geq 90 \mathrm{mmHg}$; proteinuria as a protein excretion of at least $\geq 1+$ proteinuria by dipstick in the absence of urinary tract infection. FGR was defined as a birth weight less than the 10th percentile of a reference group [20].

The data of the clinical measurement series were logarithmically transformed for the statistical analysis with ANOVA and $t$-test because they were not normally distributed. The distribution of the single parameters in the different groups was calculated by one-factorial ANOVA when comparing several groups and when comparing two groups by $t$-test for continuous parameters and Fisher-test for qualitative data. Statistical analysis was performed using the Statistical Package for Social Sciences 20 for Windows (SPSS Inc., Armonk, NY). Statistical significance was assumed at $p<0.05$. Logistic regression was used to perform univariate and multivariate analysis. Receiver operator characteristic (ROC) - curves were constructed and the area under the curves (AUC) used to compare the performance of various predictive tests.

\section{Results}

This prospective observational study enrolled 346 pregnant women. About 34 of them (9.8\%) developed PE, 26 (7.5\%) FGR and 14 (4.0\%) both pathologies (PE \& FGR). Controls $(n=272)$ included women with otherwise uncomplicated course of pregnancy. Before the second examination there occurred one intrauterine and one perinatal death during normal pregnancy course as well as one perinatal death and one late abortion in the group of PE \& IUGR.

According to final outcome, maternal characteristics at recruitment and pregnancy outcomes are shown in Table 1. There were significant differences between pre-pregnancy BMI and a previous PE or chronic hypertension between patients with either FGR or PE \& FGR compared to controls. Birthweight and GA at delivery were significantly lower in patients with FGR or PE \& FGR than in the controls (Table 1).

Table 2 presents the UAD findings and laboratory results according to the pregnancy outcome. In the second trimester, there were significant differences between the groups of FGR and PE \& FGR versus controls for the UA PI, PLGF concentration and sFlt-1/PLGF ratio. Differences were highest for PE \& FGR versus controls, while differences for pregnancies with $\mathrm{PE}$ compared to controls were insignificant (Table 2). In the third trimester, all tested variables were significantly different for the group of PE \& FGR versus controls. Pregnancies that developed PE demonstrated significantly lower PLGF levels and higher sFlt- 1 concentration and sFlt-1/PLGF ratio than controls. For pregnancies with FGR, only the differences for PLGF and the sFlt-1/PLGF quotient gained significance. As in the second trimester, all tested variables demonstrated the most prominent alterations in the group of PE \& FGR (Table 2).

Table 3 shows the prediction of PE and FGR by clinical risk factors, angiogenic biomarkers and UAD. Results are expressed as c index (area under the curve [AUC]), sensitivity and specificity as well as ROC-curves (Figures 1 and 2). Because of the small number of pregnancies with PE and FGR, analyses were performed for the groups of PE $(n=48)$ and FGR $(n=40)$, regardless of whether the women suffered from both entities at the same time. The combination of $\mathrm{UAD}$ and angiogenic factors resulted in an enlargement of the AUC compared to each parameter alone, both for PE as 
Table 1. Prenatal characteristics and maternal and fetal outcome in pregnancies with PE. FGR and PE \& FGR compared to the controls.

\begin{tabular}{lcccc}
\hline Characteristics & Controls $(n=272)$ & PE $(n=34)$ & FGR $(n=26)$ & PE \& FGR $(n=14)$ \\
\hline Maternal age, years & $24(14-41)$ & $25(14-41)$ & $23(14-39)$ & $28(19-44)^{*}$ \\
Pre-pregnancy BMI, kg/m ${ }^{2}$ & $22.7 \pm 2$ & $24.4 \pm 4^{*}$ & $22.3 \pm 1$ & $24.3 \pm 2^{*}$ \\
Primiparae, $\%(n)$ & $48(130)$ & $53(18)$ & $62(16)$ & $36(5)$ \\
Previous PE, \% $(n)$ & $5(13)$ & $29(10)^{*}$ & $4(1)$ & $29(4)^{*}$ \\
Chronic hypertension, $\%(n)$ & 0 & $6(2)^{*}$ & 0 & $7(1)^{*}$ \\
Systemic lupus erythematosus, $\%(n)$ & 0 & 0 & 0 & $14(2)^{*}$ \\
Mean GA at first visit, wks (range) & $22(18-25)$ & $22(18-25)$ & $21(19-24)$ & $22(18-25)$ \\
Mean GA at second visit, wks (range) & $30(28-33)$ & $29(28-31)$ & $30(28-31)$ & $29(28-30)$ \\
Mean GA at delivery, wks (range) & $39(26-42)$ & $38(33-40)$ & $38(33-41)^{*}$ & $36(23-41)^{*}$ \\
Birthweight, g & $3102 \pm 400$ & $2981 \pm 426$ & $2410 \pm 303^{*}$ & $2063 \pm 654^{*}$ \\
SBP at delivery, mmHg & $111 \pm 10$ & $142 \pm 8^{*}$ & $108 \pm 13$ & $149 \pm 12^{*}$ \\
DBP at delivery, mmHg & $73 \pm 8$ & $94 \pm 6^{*}$ & $72 \pm 10$ & $100 \pm 7^{*}$ \\
Proteinuria at delivery, g/l & $0.1 \pm 0.5$ & $1.5 \pm 0.7^{*}$ & $0.2 \pm 0.8$ & $1.9 \pm 0.8^{*}$ \\
\hline
\end{tabular}

PE, pre-eclampsia; FGR, fetal growth restriction; BMI, body mass index; GA, gestational age; wks, weeks; SBP, systolic blood pressure; DBP, diastolic blood pressure.

Values are given as mean \pm SEM and mean (range).

$* p<0.05$ versus controls.

Table 2. Uterine artery Doppler and laboratory results in pregnancies with PE, FGR and PE \& FGR compared to the controls in the second and third trimester.

\begin{tabular}{|c|c|c|c|c|}
\hline & Controls & PE & FGR & PE \& FGR \\
\hline PI UAD & $0.9 \pm 0.3(0.4-2)$ & $1 \pm 0.4(0.5-1.8)$ & $1.1 \pm 0.5(0.5-2.3)^{*}$ & $1.3 \pm 0.5(0.6-2)^{*}$ \\
\hline sFlt- $1, \mathrm{pg} / \mathrm{ml}$ & $1888 \pm 908(483-7534)$ & $1938 \pm 1252(225-7280)$ & $1981 \pm 706(661-4012)$ & $2158 \pm 1308(614-5427)$ \\
\hline sFlt-1/PLGF ratio & $5.5 \pm 3.5(0.6-21.7)$ & $7.1 \pm 6.3(0.8-34.7)$ & $8.7 \pm 4.8(3.5-21)^{*}$ & $10.2 \pm 6.5(2-25.8)^{*}$ \\
\hline Bilateral notch, \% ( $n)$ & $0.4(1)$ & 0 & $8(2)^{*}$ & $7(1)^{*}$ \\
\hline PLGF, pg/ml & $790 \pm 449(183-3209)$ & $414 \pm 241(162-1252)^{*}$ & $498 \pm 351(72-1529)^{*}$ & $185 \pm 123(91-439)^{*}$ \\
\hline sFlt-1, pg/ml & $1808 \pm 726(672-5613)$ & $2290 \pm 1332(821-6560)^{*}$ & $1979 \pm 701(1089-3956)$ & $5109 \pm 5438(1010-20300)^{*}$ \\
\hline sFlt-1/PLGF ratio & $2.9 \pm 1.8(0.6-10.4)$ & $7.6 \pm 6.2(1.4-29.8)^{*}$ & $6.7 \pm 6.3(1-29)^{*}$ & $44.3 \pm 58.5(3.9-203)^{*}$ \\
\hline
\end{tabular}

PE, pre-eclampsia; FGR, fetal growth restriction; wks, weeks; PI, mean pulsatility index; UAD, uterine artery Doppler.

Values are given as mean \pm SEM and mean (range).

$* p<0.05$ versus control group.

Table 3. Summary of clinical risk factors, angiogenic biomarkers and uterine artery Doppler to predict PE and FGR independently.

\begin{tabular}{|c|c|c|c|c|c|c|c|c|}
\hline & \multicolumn{4}{|c|}{$\mathrm{PE}(n=48)$} & \multicolumn{4}{|c|}{ FGR $(n=40)$} \\
\hline & $\mathrm{C}$ index & Cutoff & Sens & Spec & $\mathrm{C}$ index & Cutoff & Sens & Spec \\
\hline \multicolumn{9}{|l|}{$18-25$ wks } \\
\hline PI UAD & 0.63 & 0.9 & 0.56 & 0.7 & 0.67 & 1 & 0.63 & 0.75 \\
\hline sFlt-1/PLGF ratio & 0.6 & 6.4 & 0.54 & 0.68 & 0.72 & 4.9 & 0.88 & 0.52 \\
\hline UAD + sFlt-1/PLGF ratio & 0.66 & 0.14 & 0.65 & 0.70 & 0.77 & 0.1 & 0.75 & 0.72 \\
\hline Multivariate analysis & 0.85 & 0.11 & 0.79 & 0.78 & 0.79 & 0.1 & 0.83 & 0.64 \\
\hline \multicolumn{9}{|l|}{$28-32 \mathrm{wks}$} \\
\hline PI UAD & 0.61 & 0.9 & 0.38 & 0.83 & 0.63 & 0.9 & 0.41 & 0.85 \\
\hline sFlt-1/PLGF ratio & 0.82 & 3.5 & 0.85 & 0.71 & 0.78 & 3.9 & 0.72 & 0.74 \\
\hline UAD + sFlt-1/PLGF ratio & 0.83 & 0.1 & 0.79 & 0.75 & 0.80 & 0.09 & 0.69 & 0.77 \\
\hline Multivariate analysis & 0.89 & 0.11 & 0.83 & 0.81 & 0.77 & 0.06 & 0.64 & 0.8 \\
\hline
\end{tabular}

PE, pre-eclampsia; C index, area under the curve; Sens, sensitivity; Spec, specificity; UAD, uterine artery Doppler.

well as for FGR. The inclusion of anamnestic risk factors in the multivariate analysis led to a further improvement in the prediction of PE and FGR with an AUC of 0.85 and 0.79 in the second and 0.89 and 0.77 in the third trimester, respectively (Table 3 ).
The multivariate analysis permits the calculation of an individual risk score for PE and FGR in the clinical routine in Ecuador using the following formulas:

- Risk estimate for PE in the second trimester: $-14.3+0.5 \times$ pre-pregnancy $\mathrm{BMI}+(-0.8) \times$ deliveries 
(A)

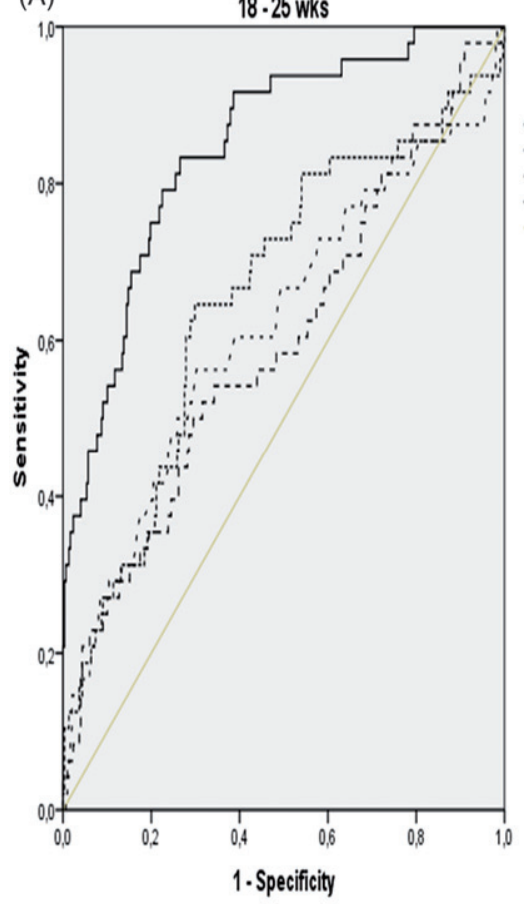

(B)

$\cdots$ UAD
$\cdots$ SPI.IPLGF ratio -... UAD + SFl-1PL.GF ratio - Mutivariate analysis - Reference ine

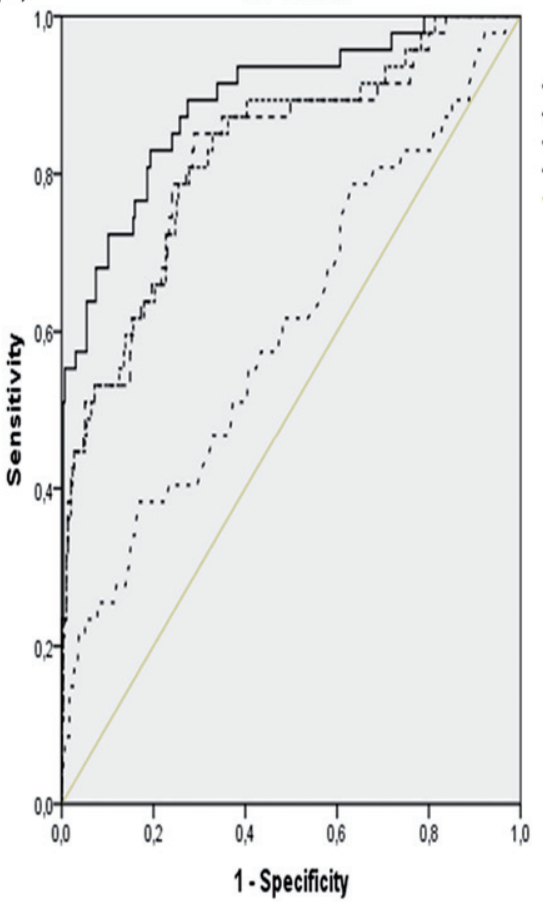

$\cdots$ VAD

- - sfl-1PLGF ratio

-... UAD + SFT. IPLGF ratio

-Mutivariate analysis

- Reference ine

Figure 1. Receiver-operating characteristic (ROC) - curves for the prediction of PE using UAD, sFlt-1/PLGF ratio, the combination of UAD and sFlt-1/PLGF ratio and multivariate analysis in the second (A) and third (B) trimester.
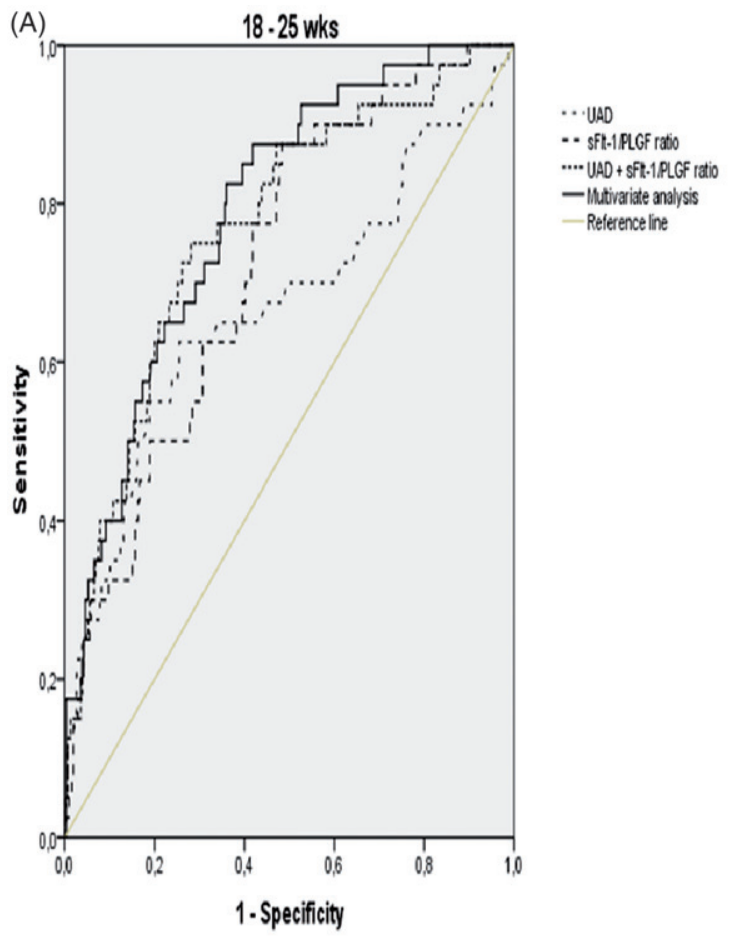

(B)

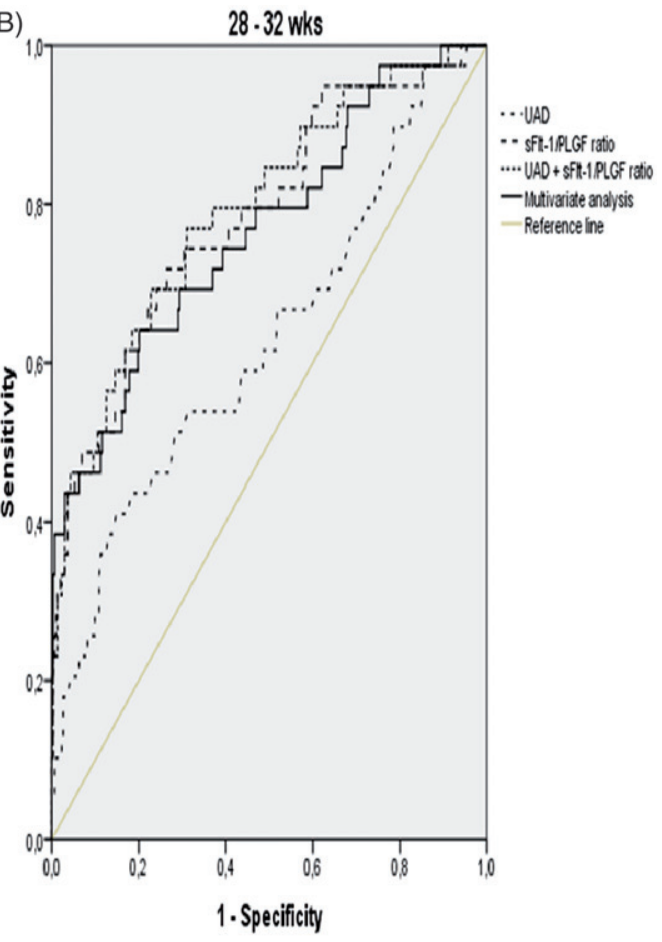

Figure 2. Receiver-operating characteristic (ROC) - curves for the prediction of FGR using UAD, sFlt-1/PLGF ratio, the combination of UAD and sFlt-1/PLGF ratio and multivariate analysis in the second (A) and third (B) trimester. 
per patient $+2.7 \times$ previous $\mathrm{PE}+1.6 \times \mathrm{UA} \mathrm{PI}+0.095 \times$ sFlt-1/PLGF ratio;

- Risk estimate for $\mathrm{PE}$ in the third trimester: $-15.5+0.5 \times$ pre-pregnancy $\mathrm{BMI}+1.9 \times$ primigravidae $+2.8 \times$ previous $\mathrm{PE}+0.3 \times$ sFlt $-1 / \mathrm{PLGF}$ ratio;

- Risk estimate for FGR in the second trimester: $-1.9+1.7 \times$ UA PI $+(-0.01) \times$ PLGF;

- Risk estimate for FGR in the third trimester: $-2.2+$ $3.2 \times$ bilateral notching $+(-0.001) \times$ sFlt $-1+0.3 \times$ sFlt-1/PLGF ratio.

The exponential function of the calculated value has to be divided through itself summarized with 1 . The result of the calculation can be compared to the cutoffs in Table 2.

\section{Discussion}

Our study focused on the value of angiogenic factors combined with UAD and maternal medical history in the prediction of PE and FGR, particularly in the Ecuadorian population. To the best of our knowledge, this is the first study exploring these factors in a high-altitude population. The findings of our study demonstrate that the combination of UAD and maternal characteristics could aid in the identification of women at risk for developing PE and FGR. This is of particular interest in a population with limited medical resources in order to organize appropriate antenatal care.

In agreement with previous studies [15,16,21,22], we found a significantly higher UA PI in the second trimester in pregnancies that developed either FGR or PE and FGR. Concerning biomarkers, PLGF values were significantly decreased and sFlt-1/PLGF ratio was increased in those with FGR as well as PE and FGR, but not in pregnancies with PE. Our inability to detect differences at 18-25 weeks between cases with $\mathrm{PE}$ and controls could be attributed to the rather low occurrence of early onset PE in our collective. Although the observed overall rate of PE was $9.8 \%$, only $1.7 \%$ developed symptoms $<34$ weeks. It has been demonstrated, that differences achieve more significance in women who developed severe, early onset disease [2,11,15,22-24]. Crispi et al. found in late-onset PE milder changes in sFlt-1 and PLGF levels than in early-onset PE with considerable overlap to controls. They made the observation that in those with late PE and abnormal UAD parameters, angiogenic placental proteins were similar to those observed in cases with early onset PE, while in the other subset with normal UAD parameters, angiogenic placental factors were similar to control subjects. Therefore, they postulated a subgroup of late-onset PE with minimal placental involvement [24].

In our study, sFlt-1 levels were not distinctive between pregnancies with normal and adverse outcome in the second trimester. The findings of a rather limited role of sFlt-1 as a single second trimester parameter are in agreement with previous studies [22,25,26]. Recently, Romero et al. investigated longitudinal changes of angiogenic factors in normal pregnancies compared to pregnancies with $\mathrm{PE}$ or small for gestational age (SGA). Comparable to our findings in FGR cases, there were no differences in plasma concentration of sFlt-1 throughout gestation between uncomplicated pregnancies and those who delivered SGA neonates. In their study, PLGF levels were decreased throughout gestation in all pregnancies destined to adverse outcome, while sFlt-1 levels became elevated only at 7 weeks prior to the diagnosis of preterm $\mathrm{PE}$ and at 11 weeks prior to the diagnosis of term PE [26].

In agreement with others, we found higher alterations in PLGF and sFlt-1 levels for the group affected by PE and FGR than for isolated PE or FGR [2]. In addition, the sFlt-1/PLGF ratio was strongest affected in pregnancies destined to develop both, PE and FGR. The observation of high sFlt-1/PLGF values not only in PE but also in FGR concurs with previous reports [21]. Like others, we found insignificant differences in sFlt-1/PLGF values between the group destined to develop PE and normal pregnancies in the second trimester [26,27].

Different cutoffs for the sFlt-1/PLGF ratio have been suggested to provide the greatest diagnostic accuracy [5]. In one study, a single cutoff of 85 for the sFlt-1/PLGF ratio was found to have a high degree of accuracy for diagnosis of PE throughout gestation, with a sensitivity of $82 \%$ and a specificity of $95 \%$. For the subgroup early-onset PE the same cutoff value resulted in a sensitivity of $89 \%$ and a specificity of $97 \%$, while for late-onset $\mathrm{PE}$ the reported sensitivity and specificity were $74 \%$ and $89 \%$, respectively [11]. More recently, the use of multiple cutoffs for the early and late gestational phase has been suggested to improve the diagnostic accuracy for PE [28]. Compared to Verlohren et al., we used a lower cutoff and observed a lower sensitivity and specificity for the diagnosis of PE. The inconsistency of these results among several studies may be a consequence of the different gestational timing of the sample collection, but also a result of different prevalence of disease in the study population. Furthermore, there is large heterogeneity in the severity and the time of onset of the disease.

Chronic hypoxia of residents of high altitude $(\geq 2500 \mathrm{~m})$ has been postulated as a key mechanism of impaired placentation, leading to maternal endothelial cell dysfunction [29]. It has been speculated that high-altitude pregnancies would have higher levels of antiangiogenic relative to proangiogenic factors compared with low-altitude pregnancies [30-32] and that this could contribute to diminished fetal growth and an increased frequency of PE. However, this hypothesis was not confirmed in previous studies, demonstrating comparable levels of PLGF and sFlt-1 in low- and high-altitude pregnancies [31,32]. In one small study, contrary to expectations, lower sFlt-1 concentrations were observed at high altitude [30]. Julian et al. investigated women in Colorado residing at low $(1600 \mathrm{~m})$ or high altitude $(3100 \mathrm{~m})$ during pregnancy $(20,30$ and 36 weeks). Altitude did not affect sFlt-1, PLGF or sFlt-1/PLGF ratio at any time. However, they observed lower nitric oxide metabolites and lower UA blood flow in high-altitude residents and this finding was associated with reduction in fetal growth [31]. These findings suggest that angiogenetic factors per se are not different in low- and high-altitude populations and play a correlative rather than a causal role in PE and FGR. Interestingly, Krampl et al. [33] found lower UA PI and RI at high altitude compared to sea level. Their normative data for high-altitude pregnancies compare well with our findings for the UA PI at 20 and 30 weeks. Despite the lower uteroplacental impedance at sea level, Krampl et al. observed significantly lower birth weights in high-altitude infants. 
Therefore, impaired placentation seems rather unlikely to be the primary cause for the reduced birth weight. The lower impedance at high altitude could be explained through more efficient early vascular remodeling of the spiral arteries as a response to lower oxygen tension [33].

A systematic review on biomarkers for the prediction of PE found a considerable amount of heterogeneity between studies, explaining the wide range of test accuracies [34]. Kleinrouweler et al. found modest but significantly different sFlt-1 and PLGF concentrations before 30 weeks in women who subsequently developed PE. Although they found their single application to be insufficient in the prediction of $\mathrm{PE}$ in clinical practice, biomarkers might be more promising when incorporated in multivariable prediction models. Combination of patient factors such as age, ethnicity, body mass index or family history of PE with biomarkers resulted in better prediction of adverse pregnancy outcome [23,27,34].

In our study, the combination of angiogenic factors with UA PI resulted in a larger AUC than for any single parameter. The inclusion of anamnestic factors in the algorithm further improved the prediction of PE and/or FGR. By customizing the indicated formulas in our study, it is possible to determine patient-specific risks for the development of PE or FGR.

Currently, there is increasing evidence that PE and delivery of small for gestational age (SGA) neonates can be predicted at 11-13 weeks' gestation by a combination of maternal medical and obstetric history, UA PI, mean arterial pressure and maternal serum biochemical markers [35-37]. Although several biochemical markers have been proposed, PAPP-A and PlGF are the only two that have been shown to be useful in first trimester screening, while sFlt- 1 and the sFlt-1/PLGF ratio show a low predictive value $[5,26,38]$. The combined screening using maternal characteristics, biophysical and biochemical factors resulted in detection rates of early-PE, late-PE, preterm-SGA and term-SGA of $95.3 \%, 47.1 \%, 52.6 \%$ and $42.1 \%$ at a false-positive rate of $10 \%$, respectively [37].

We acknowledge some limitations of the study. First of all, the small sample size as well as the associated numbers of early PE and FGR. Furthermore, there is huge diversity of ethnicities and socio-economic groups in Ecuador, therefore our results might not be applicable to the complete Ecuadorian population.

In conclusion, this study reveals that screening by angiogenic factors and uterine PI, especially when combined with clinical features, may contribute to a prediction of PE and FGR in the Ecuadorian population. An early detection of any of those pregnancy complications may be essential to offer an optimal monitoring and supportive care to mothers and fetuses at risk. Further studies with larger study groups and different subgroups associated with PE and FGR are desirable in order to find additional support for our results.

\section{Acknowledgements}

We would like to thank Dr. Humberto Navas, Dr. Ricardo Carrillo, Dr. Iván Ruilova, Dr. Rolando Montesinos and Silvia Cóndor, from the Obstetrics and Gynecology Hospital Isidro Ayora in Quito, Ecuador; Dr. Mauricio Corral, Department of Gynecology and Obstetrics, Hospital Metropolitano, Quito, Ecuador; Dr. Luis Narvaez and Dr. Marcelo Cruz, Netlab,
Quito, Ecuador; Carlos Fiallos and Dra. Malena Tapia, Roche, Quito, Ecuador and Dr. Wim van der Helm, Roche, Switzerland for providing support to Carolin Kienast during the realization of the study in Ecuador.

\section{Declaration of interest}

The test kits were given as a free grant by Roche Diagnostics, Switzerland. O.R. is employed by Roche, Ecuador. Dr. Rolf Fimmers and Dr. Ute Klarmann, Department of Medical Biometrics, Epidemiology and Informatics, University Bonn, for providing support in the statistical analyses. None of the other authors has a conflict of interest.

\section{References}

1. Steegers EA, von Dadelszen P, Duvekot JJ, Pijnenborg R. Preeclampsia. Lancet 2010;376:631-44.

2. Levine RJ, Maynard SE, Qian C, et al. Circulating angiogenic factors and the risk of preeclampsia. N Engl J Med 2004;350: 672-83.

3. Lam C, Lim KH, Karumanchi SA. Circulating angiogenic factors in the pathogenesis and prediction of preeclampsia. Hypertension 2005;46:1077-85.

4. Kinzler WL, Vintzileos AM. Fetal growth restriction: a modern approach. Curr Opin Obstet Gynecol 2008;20:125-31.

5. Lapaire O, Shennan A, Stepan H. The preeclampsia biomarkers soluble fms-like tyrosine kinase-1 and placental growth factor: current knowledge, clinical implications and future application. Eur J Obstet Gynecol Reprod Biol 2010;151:122-9.

6. De Vivo A, Baviera G, Giordano D, et al. Endoglin, PLGF and sFlt1 as markers for predicting pre-eclampsia. Acta Obstet Gynecol Scand 2008;87:837-42.

7. Hertig A, Berkane N, Lefevre G. Maternal serum sFlt-1 concentration is an early and reliable predictive marker of preeclampsia. Clin Chem 2004;50:1702-3.

8. McKeeman GC, Ardill JE, Caldwell CM, et al. Soluble vascular endothelial growth factor receptor-1 (sFlt-1) is increased throughout gestation in patients who have preeclampsia develop. Am J Obstet Gynecol 2004;191:1240-6.

9. Taylor RN, Grimwood J, Taylor RS, et al. Longitudinal serum concentrations of placental growth factor: evidence for abnormal placental angiogenesis in pathologic pregnancies. Am J Obstet Gynecol 2003;188:177-82.

10. Tjoa ML, van Vugt JM, Mulders MA, et al. Plasma placenta growth factor levels in midtrimester pregnancies. Obstet Gynecol 2001;98: $600-7$.

11. Verlohren S, Galindo A, Schlembach D, et al. An automated method for the determination of the sFlt-1/PLGF ratio in the assessment of preeclampsia. Am J Obstet Gynecol 2010;202: 161e1-11.

12. Gómez O, Martínez JM, Figueras F, et al. Uterine artery Doppler at 11-14 weeks of gestation to screen for hypertensive disorders and associated complications in an unselected population. Ultrasound Obstet Gynecol 2005;26:490-4.

13. Albaiges G, Missfelder-Lobos H, Lees C, et al. One-stage screening for pregnancy complications by color Doppler assessment of the uterine arteries at 23 weeks' gestation. Obstet Gynecol 2000;96: 559-64.

14. Bower S, Bewley S, Campbell S. Improved prediction of preeclampsia by two stage screening of uterine arteries using the early diastolic notch and color Doppler imaging. Obstet Gynecol 1993;82:78-83.

15. Stepan H, Unversucht A, Wessel N, Faber R. Predictive value of maternal angiogenic factors in second trimester pregnancies with abnormal uterine perfusion. JAHA 2007;49:818-24.

16. Diab AE, El-Behery MM, Ebrahiem MA, Shehata AE. Angiogenic factors for the prediction of pre-eclampsia in women with abnormal midtrimester uterine artery Doppler velocimetry. Int J Gynaecol Obstet 2008;102:146-51.

17. Duley L. The global impact of pre-eclampsia and eclampsia. Semin Perinatol 2009;33:130-7. 
18. Escudero C, Calle A. Hierro, oxígeno y desarrollo placentario en la génesis de la preeclampsia. Efectos de la altura en Ecuador. Rev Méd Chile 2006;134:491-8.

19. Keyes LE, Armaza JF, Niermeyer S, et al. Intrauterine growth restriction, preeclampsia and intrauterine mortality at high altitude in Bolivia. Pediatr Res 2003;54:20-5.

20. Mikolajczyk RT, Zhang J, Betran AP, et al. A global reference for fetal-weight and birthweight percentiles. Lancet 2011;377: $1855-61$.

21. Gómez-Arriaga PI, Herraiz I, López-Jiménez EA, et al. Uterine artery Doppler and sFlt-1/PLGF ratio: usefulness in diagnosis of pre-eclampsia. Ultrasound Obstet Gynecol 2013;41:530-7.

22. Espinoza J, Romero R, Nien JK, et al. Identification of patients at risk for early onset and/or severe preeclampsia with the use of uterine artery Doppler velocimetry and placental growth factor. Am J Obstet Gynecol 2007;196:326.e1-13.

23. Widmer M, Villar J, Benigni A, et al. Mapping the theories of preeclampsia and the role of angiogenic factors: a systematic review. Obstet Gynecol 2007;109:168-80.

24. Crispi F, Domínguez C, Llurba E, et al. Placental angiogenic growth factors and uterine artery Doppler findings for characterization of different subsets in preeclampsia and in isolated intrauterine growth restriction. Am J Obstet Gynecol 2006;195: 201-7.

25. Myers JE, Kenny LC, McCowan LM, et al. Angiogenic factors combined with clinical risk factors to predict preterm pre-eclampsia in nulliparous women: a predictive test accuracy study. BJOG 2013; 120:1215-23.

26. Romero R, Nien JK, Espinoza J, et al. A longitudinal study of angiogenic (placental growth factor) and anti-angiogenic (soluble endogen and soluble vascular endothelial growth factor receptor-1) factors in normal pregnancy and patients destined to develop preeclampsia and deliver a small for gestational age neonate. J Matern Fetal Neonatal Med 2008;21:9-23.

27. Diguisto C, Le Gouge A, Piver E, et al. Second-trimester uterine artery Doppler, PLGF, sFlt-1, sEndoglin, and lipid-related markers for predicting preeclampsia in a high-risk population. Prenat Diagn 2013;33:1070-4.
28. Verlohren S, Herraiz I, Lapaire O, et al. New gestational phasespecific cutoff values for the use of the soluble fms-like tyrosine kinase-1/placental growth factor ratio as a diagnostic test for preeclampsia. Hypertension 2014;63:346-52.

29. Young BC, Levine RJ, Karumanchi SA. Pathogenesis of preeclampsia. Annu Rev Pathol 2010;5:173-92.

30. Dávila RD, Julian CG, Wilson MJ, et al. Do anti-angiogenic or angiogenic factors contribute to the protection of birth weight at high altitude afforded by Andean ancestry? Reprod Sci 2010;17: 861-70.

31. Julian CG, Galan HL, Wilson MJ, et al. Lower uterine artery blood flow and higher endothelin relative to nitric oxide metabolite levels are associated with reductions in birth weight at high altitude. Am J Physiol Regul Integr Comp Physiol 2008;295:R906-15.

32. Zamudio S, Borges M, Echalar L, et al. Maternal and fetoplacental hypoxia do not alter circulating angiogenic growth effectors during human pregnancy. Biol Reprod 2014;90:42.

33. Krampl E, Lees C, Bland JM, et al. Fetal Doppler velocimetry at high altitude. Ultrasound Obstet Gynecol 2001;18:329-34.

34. Kleinrouweler CE, Wiegerinck MM, Ris-Stalpers C, et al. Accuracy of circulating placental growth factor, vascular endothelial growth factor, soluble fms-like tyrosine kinase 1 and soluble endoglin in the prediction of pre-eclampsia: a systematic review and meta-analysis. BJOG 2012;119:778-87.

35. Akolekar R, Syngelaki A, Sarquis R, et al. Prediction of early, intermediate and late pre-eclampsia from maternal factors, biophysical and biochemical markers at 11-13 weeks. Prenat Diagn 2011;31:66-74.

36. Karagiannis G, Akolekar R, Sarquis R, et al. Prediction of smallfor-gestation neonates from biophysical and biochemical markers at 11-13 weeks. Fetal Diagn Ther 2011;29:148-54.

37. Poon L, Syngelaki A, Akolekar R, et al. Combined screening for preeclampsia and small for gestational age at 11-13 weeks. Fetal Diagn Ther 2013;33:16-27.

38. Parra-Cordero M, Rodrigo R, Barja $\mathrm{P}$, et al. Prediction of early and late pre-eclampsia from maternal characteristics, uterine artery Doppler and markers of vasculogenesis during first trimester of pregnancy. Ultrasound Obstet Gynecol 2013;41: $538-44$. 